\title{
High metabolic tumor volume and total lesion glycolysis are associated with lateral lymph node metastasis in patients with incidentally detected thyroid carcinoma
}

\author{
Bo Hyun Kim ${ }^{1}$ - Seong-Jang Kim ${ }^{2}$ Keunyoung Kim ${ }^{2}$ - Heeyoung Kim ${ }^{2}$. \\ So Jung Kim ${ }^{2}$ - Won Jin Kim ${ }^{1}$ - Yun Kyung Jeon ${ }^{1}$ Sang Soo Kim ${ }^{1}$ • \\ Yong Ki Kim ${ }^{3}$ In Joo Kim ${ }^{1}$
}

Received: 26 February 2015/Accepted: 17 June 2015/Published online: 25 June 2015

(C) The Japanese Society of Nuclear Medicine 2015

\begin{abstract}
Objective The objective of this study was to investigate whether total lesion glycolysis (TLG) and metabolic tumor volume (MTV) measured by ${ }^{18} \mathrm{~F}$-fluorodeoxyglucose $\left({ }^{18} \mathrm{~F}\right.$ FDG) positron emission tomography/computed tomography (PET/CT) could predict the aggressiveness and lymph node metastasis (LNM) in patients with incidentally detected differentiated thyroid carcinoma.

Methods A total 358 patients with focal FDG-avid thyroid incidentaloma during cancer evaluation were enrolled. Among 235 patients in whom fine-needle aspiration biopsy was performed, 51 patients underwent total thyroidectomy with $\mathrm{LN}$ dissection. We analyzed the relationship between volume-based parameters and clinicopathologic characteristics.

Results The mean age and tumor size were $57.1 \pm$ 11.3 years and $1.15 \pm 0.81 \mathrm{~cm}$, respectively. The prevalence
\end{abstract}

Seong-Jang Kim

growthkim@daum.net; growthkim@pusan.ac.kr

Bo Hyun Kim

pons71@hanmail.net

Keunyoung Kim

4mura2@hanmail.net

Heeyoung Kim

dinggury84@hanmail.net

So Jung Kim

laocone@naver.com

Won Jin Kim

bearshow00@hanmail.net

Yun Kyung Jeon

puritystar@hanmail.net

Sang Soo Kim

drsskim7@gmail.com of malignancy was $21.7 \%(51 / 235)$. When $\mathrm{SUV}_{\max }>5.91$, MTV2.5 $>2.05 \mathrm{~cm}^{3}$, and TLG2.5> 9.09 were used as cutoff points, sensitivity, specificity, and area under curve (AUC) for prediction of lateral LNM were 77.9, 69.1\%, 0.716 ( $P=0.047), 77.8,88.1 \%, 0.839$ ( $P<0.001), 77.8,85.1 \%$, and $0.815(P=0.002)$, respectively. However, MTV and TLG had no value in prediction of central LNM, extrathyroidal extension, and multifocality. On comparison ROC curve analysis, the MTV and TLG showed the statistical differences for the prediction of lateral LNM compared with $\mathrm{SUV}_{\text {max }}$ (all $P$ 's $<0.05$ ).

Conclusions This study has shown for the first time that volume-based PET functional parameters had a significant value for the prediction of lateral LNM in incidentally detected PTC. These results suggest that higher MTV and TLG can be potential new risk factors for preoperative risk stratification. The usefulness of TLG and MTV in

Yong Ki Kim

yongki@pusan.ac.kr

In Joo Kim

injkim@pusan.ac.kr

1 Department of Internal Medicine, School of Medicine, Pusan National University and Biomedical Research Institute, 179 Gudeok-Ro, Seo-Gu, Busan 602-739, Korea

2 Department of Nuclear Medicine, School of Medicine, Pusan National University and Biomedical Research Institute, 179 Gudeok-Ro, Seo-Gu, Busan 602-739, Korea

3 Kim Yong Ki Internal Medicine Clinic, Chungmu-dong 1-ga, Seo-gu, Busan 602-011, Korea 
preoperative risk stratification in patients with PTC needs to be confirmed in further large studies.

Keywords Metabolic tumor volume - Total lesion glycolysis · Papillary thyroid carcinoma $\cdot$ Lymphatic metastasis

\section{Introduction}

Fluorodeoxyglucose labeled with ${ }^{18}$ Fluorine $\left({ }^{18} \mathrm{~F}-\mathrm{FDG}\right)$ positron emission tomography/computed tomography (PET/CT) has an advantage over conventional modalities such as CT or magnetic resonance imaging (MRI) in that it enables quantification of the metabolic activity of a tumor. Functional imaging of FDG PET/CT can provide metabolic information on malignant tissues and make it possible to reflect the tumor burden more accurately [1]. Therefore, volume-based PET parameters such as metabolic tumor volume (MTV) and total lesion glycolysis (TLG) have been developed to measure metabolic activity in an entire tumor mass [1,2].

MTV is a semi-quantitative parameter of metabolic activity of tumors determined by ${ }^{18} \mathrm{~F}$-FDG PET/CT images. MTV potentially has clinical value in the evaluation of tumor biology, evaluation of response to treatment, and prognostication in various cancers [3-6]. As another semiquantitative parameter, TLG is called the Larson-Ginsberg index, which was calculated by multiplying the mean standardized uptake value $\left(\mathrm{SUV}_{\text {mean }}\right)$ by the MTV. TLG corresponds to the cell mass of the target lesion associated with FDG uptake. TLG has been suggested to better reflect global metabolic activity in whole tumors [1]. Thus, TLG have the potential to become valuable imaging biomarkers in human solid tumors as prognostic biomarkers, adding value to clinical staging, for treatment response assessment and for treatment optimization [7].

Preoperative detection of lateral lymph node metastasis (LNM) in differentiated thyroid carcinoma (DTC) was very important for surgical plan. In addition, lateral LNM of DTC may reflect the biological aggressiveness of the carcinoma [8]. LNM may be preoperatively evaluated by imaging tests including ultrasonography (US), CT, magnetic resonance imaging (MRI), and PET/CT. Although US is the most routinely recommended of these imaging methods for preoperative evaluation of LNM in patients with papillary thyroid carcinoma (PTC) [9, 10], no other study has attempted to adapt volume-based PET parameters such as TLG and MTV for prediction of lateral LNM and characterization of DTC. The objective of this study was to investigate whether TLG and MTV measured by ${ }^{18}$ F-FDG PET/CT could predict the aggressiveness and lateral LNM in patients with incidentally detected DTC.

\section{Materials and methods}

\section{Patients}

From August 2010 to April 2013, 18,172 subjects (7589 men and 10583 women) with known or suspected cancer and healthy people in Pusan National University Hospital were enrolled in the current study. The purpose of ${ }^{18} \mathrm{~F}$ FDG PET/CT scan was as follows: initial staging of the cancer in 9064, treatment response determination in 5186 , detection of recurrent and metastatic diseases in 3306 , evaluation for cancer of unknown primary origin in 383 , and screening for health checkup in 233 subjects. This study was approved by the Institutional Review Board of Pusan National University Hospital, and all subjects gave their informed consent to participate in the study.

Three hundred fifty eight patients (374 nodules) have focal FDG-avid thyroid nodule in ${ }^{18}$ F-FDG PET/CT scan. Among these 358 patients, cytological diagnosis with fineneedle aspiration biopsy (FNAB) was performed in 235 patients. The results of FNAB were 36 malignant, 12 suspicious malignant, 4 follicular neoplasm, 13 atypia of undetermined significance (AUS), 153 benign, and 17 nondiagnostic. However, 101 patients did not receive FNAB due to underlying cancer, general condition, and follow-up loss. In addition, US was performed in 22 patients without FNAB due to typical benign ultrasonographic features. All 48 patients with FNAB results of malignant or suspicious malignant who underwent total thyroidectomy and central lymph node dissection were surgically confirmed as PTC. Two AUS nodules and one follicular neoplasm were confirmed as PTC and minimally invasive follicular thyroid carcinoma (FTC) by surgery, respectively. All lateral LNM had been localized by US and confirmed by FNAB prior to surgery. Especially, thirteen patients (20 lateral LNs) have FDG-avid lateral lymph node in ${ }^{18}$ F-FDG PET/CT scan. Among these patients, 9 patients underwent lateral $\mathrm{LN}$ dissection for lateral LN metastasis confirmed by preoperative US-guided FNA with $\mathrm{Tg}$ assay. The prevalence of malignancy was $21.7 \%$ (51/235). Finally, we analyzed 51 patients in this study.

\section{${ }^{18}$ F-FDG PET-CT imaging}

${ }^{18}$ F-FDG PET/CT images were obtained with a dedicated PET-CT scanner (Biograph 40, SIEMENS, Knoxville, TN, USA). Standard patient preparation included at least 8-hour fasting and a serum glucose level of less than $6.6 \mathrm{mmol} / \mathrm{L}$ before ${ }^{18}$ F-FDG administration. PET/CT imaging was performed $60 \mathrm{~min}$ after injection of ${ }^{18} \mathrm{~F}$-FDG $(5 \mathrm{MBq}$ per kilogram of body weight). CT scan $(120 \mathrm{kV}, 30 \mathrm{~mA})$ with 
slice width of $4 \mathrm{~mm}$ was obtained before the PET acquisition for attenuation correction. Emission scan time per bed position was $3 \mathrm{~min}$; six bed positions were acquired. PET data were obtained using a high-resolution wholebody scanner with an axial field of view of $21.6 \mathrm{~cm}$. The average axial resolution varied between $2.0 \mathrm{~mm}$ full width at half maximum in the center and $2.4 \mathrm{~mm}$ at $28 \mathrm{~cm}$. The average total PET-CT examination time was $20 \mathrm{~min}$.

\section{${ }^{18}$ F-FDG PET/CT image analysis}

PET/CT data sets were evaluated by 2 nuclear physicians blinded to all imaging studies and clinical and pathologic results. Decisions concerning the analysis of ${ }^{18} \mathrm{~F}-\mathrm{FDG}$ PET/ CT data sets were reached by consensus. In ${ }^{18} \mathrm{~F}-\mathrm{FDG}$ PET/ CT images, thyroid uptake was considered to be present when there was increased uptake in the thyroid gland above physiologic background. Focal thyroid uptake was defined as FDG uptake in less than one lobe of the thyroid gland. This definition was used in an attempt to separate patients with thyroiditis such as Hashimoto's thyroiditis, which usually manifests with diffuse FDG thyroid uptake [11]. When focal FDG activity was seen in the thyroid region, the fused images and the CT images were evaluated to ensure that uptake was from within the thyroid gland. In addition, if there were multiple thyroid nodules in focal FDG uptake lesions in both thyroid glands, we evaluated the largest thyroid nodule with focal intense FDG uptake. There was no artifact from the clavicle encountered in PET/CT image.

The PET/CT data sets were analyzed semi-quantitatively by the use of the maximum standardized uptake value $\left(\mathrm{SUV}_{\max }\right)$ of ${ }^{18} \mathrm{~F}-\mathrm{FDG}$ uptake. Semi-quantitative evaluation was performed with a syngo Multimodality Workplace (MMWP) (C) Siemens AG.) The SUV max and $\mathrm{SUV}_{\text {mean }}$ were semi-quantitatively used to determine ${ }^{18} \mathrm{~F}$ FDG avidity. The SUVs were determined by the region-ofinterest technique. $\mathrm{SUV}_{\text {mean }}$ was defined as the mean tumor concentration of FDG $(\mathrm{kBq} / \mathrm{mL})$ divided by the injected dose $(\mathrm{kBq})$, corrected for patient body weight $(\mathrm{g})$. To calculate $\mathrm{SUV}_{\max }$, manually defined circular regions of interest (ROI) were drawn on the attenuation-corrected emission images throughout the axial plane in which a suspicious lesion could be delineated.

\section{Measurement of metabolic tumor volume and total lesion glycolysis on ${ }^{18}$ F-FDG PET/CT}

MTV and TLG were obtained with each threshold of SUV as $2.5,3.0,3.5$, and 4.0 .

We drew the volume of interest(VOI), which showed equal or greater than absolute SUV of 2.5, 3.0, 3.5, and 4.0 on each axial image of PET-CT. MTV was determined using a fixed background SUV cutoff, all voxels containing
SUV values above this threshold constituting the MTV. The VOI was drawn so as to include hypermetabolic thyroid nodule. The boundaries were drawn large enough to include the thyroid tumor in the PET images. The contouring margins around the target lesion of thyroid were automatically produced. The voxels presenting SUV intensity of equal or greater than each threshold of the $\mathrm{SUV}_{\text {max }}$ of the primary tumor on each axial image of PET/ CT were incorporated to define the MTV.

As previously described [12], after all hypermetabolic tumor foci were segmented, MTV was calculated by summation of all the voxels of each slice of PET/CT. If $\mathrm{SUV}_{\max }$ of the primary tumor was lower than that of the threshold, we regarded the MTV of the lesion as 0 . We did not use point spread function (PSF) reconstruction algorithm for determination of MTV in this study. TLG was calculated by multiplying the $\mathrm{SUV}_{\text {mean }}$ value of the tumor by the MTV of the tumor.

\section{Statistical analysis}

Statistical analyses were performed using commercially available software (MedCalc 12.3, Mariakerke, Belgium). Continuous data are expressed as mean \pm SD for normally distributed values and median (range) for nonparametric values (skewed variables). Categorical data were presented as frequency and percentage. Receiver operating characteristic (ROC) analyses were performed to determine the optimal cutoff values of MTV and TLG yielding the maximal sensitivity and specificity for prediction of pathologic lateral LN metastasis of thyroid cancer. ROC curves for each parameter were derived and evaluated by comparing the areas under the curve (AUCs). Patients were classified into two groups, namely those with low and high values of $\mathrm{SUV}_{\max }$, MTV, and TLG according to ROC cutoff values. Various volumetric parameters and clinicopathologic characteristics were evaluated using the Chisquare test or Fisher's exact test between lateral LN positive and negative, as appropriate. To compare the semiquantitative indices according to tumor size, we performed Kruskal-Wallis tests. We used comparison ROC curve analysis to test the statistical significance of the difference among the parameters of F-18 FDG PET-CT. Statistical significance was defined as $P<0.05$.

\section{Results}

\section{Patient characteristics}

Patient and tumor characteristics are shown in Table 1. The mean age was $57.1 \pm 11.3$ years (range $34-88$ years). The mean tumor size was $1.15 \pm 0.81 \mathrm{~cm}$ (range $0.4-4.5$ ). The 
Table 1 Clinical characteristics of the patients

\begin{tabular}{|c|c|}
\hline Characteristics & Value \\
\hline Total number of patients & 51 \\
\hline Age, year, mean \pm SD (range) & $57.1 \pm 11.3(34-88)$ \\
\hline No. of women $(\%)$ & $45(88.2)$ \\
\hline Tumor size, $\mathrm{cm}$, mean \pm SD (range) & $1.15 \pm 0.81(0.4-4.5)$ \\
\hline $\mathrm{SUV}_{\max }$, median (range) & $5.67(1.99-52.25)$ \\
\hline MTV2.5, $\mathrm{cm}^{3}$, median (range) & $0.95(0-29.35)$ \\
\hline MTV3, $\mathrm{cm}^{3}$, median (range) & $0.72(0-26.44)$ \\
\hline MTV3.5, $\mathrm{cm}^{3}$, median (range) & $0.51(0-24.86)$ \\
\hline MTV4, $\mathrm{cm}^{3}$, median (range) & $0.39(0-23.25)$ \\
\hline TLG2.5, median (range) & $3.98(0-407.08)$ \\
\hline TLG3, median (range) & $3.34(0-398.98)$ \\
\hline TLG3.5, median (range) & $2.80(0-393.78)$ \\
\hline TLG4, median (range) & $1.99(0-387.58)$ \\
\hline Multifocality (\%) & $16(31.4)$ \\
\hline Extrathyroidal extension (\%) & $23(45.1)$ \\
\hline LN metastasis $(\%)$ & $26(51.0)$ \\
\hline Lateral LN metastasis (\%) & $9(17.6)$ \\
\hline Advanced stage $(\%)^{\mathrm{a}}$ & $30(62.0)$ \\
\hline
\end{tabular}

Data are expressed as mean $\pm \mathrm{SD}$ and median (range) for continuous variables and frequency (\%) for categorical variables

$S U V$ standardized uptake value, MTV metabolic tumor volume, $T L G$ total lesion glycolysis

a Advanced stage, TNM stage III and IV

proportion of patients with papillary thyroid microcarcinoma (PTMC) was $68 \%$ (34/50) with the exception of one minimally invasive FTC. According to the pathologic results, the lateral LNM was present in 9 of 51 patients $(17.6 \%)$. The proportion of advanced American Joint Committee on Cancer (AJCC) stage (stage III and IV) was $62.0 \%$ (31/50).

\section{Comparison of semi-quantitative indices according to pathologic tumor size}

Figure 1 demonstrates the differences of $\mathrm{SUV}_{\max }$ and MTV according to pathologic tumor size. The medians of $\mathrm{SUV}_{\max }$ and MTV2.5 were significantly higher in patients who had a tumor greater than $1.5 \mathrm{~cm}$ in size as compared to those who had a tumor $\leq 1.5 \mathrm{~cm}$ in size $(P<0.001)$.

\section{Association of FDG uptake of lateral lymph node and FNA results of lateral lymph node}

Table 2 shows the association between FDG uptake of lateral LN and US-guided FNA results. Among 51 patients, twenty lateral LNs with FDG uptake were detected in 13 patients $(25.5 \%)$. The mean number of FDG uptake of lateral LN per patients was $1.54 \pm 0.66$ (range 1-3). The sensitivity, specificity, accuracy, positive predictive value (PPV), and negative predictive value (NPV) of FDG uptake for lateral LNM were 66.7, 83.0, 80.4, 46.2, and $92.1 \%$, respectively. However, three patients who had US-guided FNA-confirmed lateral LNM did not show FDG uptake in lateral LNs on F18-FDG $\mathrm{PET} / \mathrm{CT}$.

\section{ROC analyses of $\mathrm{SUV}_{\max }$ and volume-based parameters for poor pathologic characteristics}

The ability of the $\mathrm{SUV}_{\max }$, MTV, and TLG of the primary tumor to predict poor pathologic characteristics including central LNM, extrathyroidal extension (ETE), and multifocality was depicted by the ROC curve. When $\mathrm{SUV}_{\max }$ $>4.6$ was used as the cutoff point, the area under curve (AUC) for prediction of central LNM was $0.576(95 \% \mathrm{CI}$ 0.429-0.713 $) \quad(P=0.361)$. When $\mathrm{SUV}_{\max }>6.68$ was used as the cutoff point, the AUC for prediction of ETE was 0.589 (95\% CI 0.442-0.724) ( $P=0.292)$. When $\mathrm{SUV}_{\text {max }}>9.07$ was used as the cutoff point, the AUC for prediction of multifocality was $0.511 \quad(95 \%$ CI 0.367-0.653) $(P=0.909)$. The MTV had no predictive value in central LNM (AUC $=0.535, \quad P=0.679)$, ETE $\quad(\mathrm{AUC}=0.571, \quad P=0.401)$, and multifocality (AUC $=0.501, P=0.992$ ). In addition, the TLG had no predictive value in central $\mathrm{LNM}$ ( $\mathrm{AUC}=0.536$,
Fig. 1 The differences of semiquantitative parameters (a $\mathrm{SUV}_{\max }$, b MTV2.5) according to tumor size
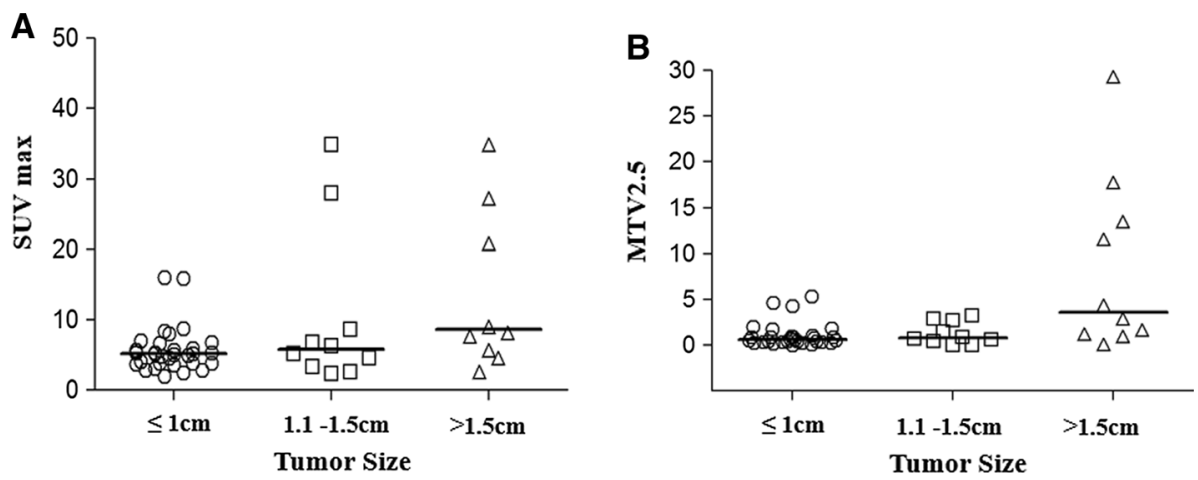
Table 2 Association of FDG uptake of lateral lymph node and ultrasonography-guided FNA results in patients with FDG positivity of lateral lymph node

\begin{tabular}{|c|c|c|c|}
\hline $\begin{array}{l}\text { Patient } \\
\text { no }\end{array}$ & $\begin{array}{l}\text { Location of lateral } \\
\text { LN }\end{array}$ & $\begin{array}{l}\mathrm{SUV}_{\max } \text { of lateral } \\
\mathrm{LN}\end{array}$ & $\begin{array}{l}\text { FNA } \\
\text { result }\end{array}$ \\
\hline 1 & Rt. II & 3.4 & Reactive \\
\hline \multirow[t]{2}{*}{2} & Lt. II & 5.3 & Metastasis \\
\hline & Lt. III & 16.0 & Metastasis \\
\hline 3 & Rt.IB & 4.7 & Metastasis \\
\hline \multirow[t]{2}{*}{4} & Lt.II & 3.5 & Reactive \\
\hline & Lt.III & 3.1 & Metastasis \\
\hline 5 & Rt.IB & 3.6 & Reactive \\
\hline 6 & Rt.III & 7.0 & Reactive \\
\hline \multirow[t]{2}{*}{7} & Rt.II & 3.8 & Reactive \\
\hline & Lt.II & 3.2 & Reactive \\
\hline 8 & Lt.V & 12.7 & Reactive \\
\hline 9 & Lt.III & 3.1 & Reactive \\
\hline \multirow[t]{2}{*}{10} & Rt.II & 6.8 & Metastasis \\
\hline & Lt.II & 4.0 & Metastasi \\
\hline 11 & Lt.II & 4.6 & Reactive \\
\hline \multirow[t]{3}{*}{12} & Rt.II & 9.2 & Metastas \\
\hline & Rt.IV & 11.6 & Metastas \\
\hline & Rt.III & 2.8 & Metastas \\
\hline \multirow[t]{2}{*}{13} & Lt.II & 10.3 & Metastas \\
\hline & Lt.III & 5.8 & Metastas \\
\hline
\end{tabular}

The classification of cervical lymph nodes from consensus statement on the terminology and classification of central neck dissection for thyroid cancer. 2009 The American Thyroid Association Surgery Working Group

$F D G$ fluorodeoxyglucose, $L N$ lymph node, $F N A$ fine-needle aspiration, $S U V$ standardized uptake value

$P=0.664)$, ETE $($ AUC $=0.607, P=0.199)$, and multifocality $(\mathrm{AUC}=0.510, P=0.914)$.

\section{Comparison of clinicopathologic characteristics and semi-quantitative metabolic parameters according to lateral lymph node metastasis}

Table 3 shows the comparison between $\mathrm{SUV}_{\max }$, MTV, TLG, and various clinical characteristics, including age, tumor size, ETE, central LNM, and multifocality according to lateral LNM. Lateral LN metastases were common in the group of tumor greater than $1 \mathrm{~cm}$ in size $(P=0.020)$ and central LNM group $(P=0.047)$. However, there was no significant difference of age $(P=0.947)$, sex $(P=0.140)$, ETE $(P=0.268)$, and multifocality $(P=0.701)$ between two groups. The lateral LNM group showed statistically significant higher values of $\operatorname{SUV}_{\text {max }}(P=0.020)$, all MTV (all $P$ 's $<0.001$ ), and all TLG (all $P$ 's $<0.001$ ) than those of the lateral LN-negative group.
Table 3 Comparison of clinicopathologic characteristics and semiquantitative metabolic parameters according to lateral lymph node metastasis

\begin{tabular}{|c|c|c|c|}
\hline \multirow[t]{2}{*}{ Variables } & \multicolumn{2}{|c|}{ Lateral lymph node metastasis } & \multirow{2}{*}{$P$} \\
\hline & Negative number $(\%)$ & Positive number $(\%)$ & \\
\hline \multicolumn{4}{|l|}{ Age (years) } \\
\hline$>45$ & $37(82.2)$ & $8(17.8)$ & \multirow[t]{2}{*}{0.947} \\
\hline$\leq 45$ & $5(83.3)$ & $1(16.7)$ & \\
\hline \multicolumn{4}{|l|}{ Sex } \\
\hline Male & $12(70.6)$ & $5(29.4)$ & \multirow[t]{2}{*}{0.140} \\
\hline Female & $30(88.2)$ & $4(11.8)$ & \\
\hline \multicolumn{4}{|c|}{ Tumor size $(\mathrm{cm})$} \\
\hline$>1$ & $13(65.0)$ & $7(35.0)$ & \multirow[t]{2}{*}{0.020} \\
\hline$\leq 1$ & $29(93.5)$ & $2(6.5)$ & \\
\hline \multicolumn{4}{|l|}{ ETE } \\
\hline Presence & 17 (73.9) & $6(26.1)$ & \multirow[t]{2}{*}{0.268} \\
\hline Absence & $25(89.3)$ & $3(10.7)$ & \\
\hline \multicolumn{4}{|l|}{ CLN } \\
\hline Presence & $17(70.8)$ & $7(29.2)$ & \multirow[t]{2}{*}{0.047} \\
\hline Absence & $25(92.6)$ & $2(7.4)$ & \\
\hline \multicolumn{4}{|c|}{ Multifocality } \\
\hline Presence & $14(87.5)$ & $2(12.5)$ & \multirow[t]{2}{*}{0.701} \\
\hline Absence & $28(80.0)$ & $7(20.0)$ & \\
\hline \multicolumn{4}{|l|}{$\mathrm{SUV}_{\max }$} \\
\hline$>5.91$ & $13(65.0)$ & $7(35.0)$ & \multirow[t]{2}{*}{0.020} \\
\hline$\leq 5.91$ & $29(93.5)$ & $2(6.5)$ & \\
\hline \multicolumn{4}{|c|}{$\operatorname{MTV} 2.5\left(\mathrm{~cm}^{3}\right)$} \\
\hline$>2.05$ & $5(41.7)$ & $7(58.3)$ & \multirow[t]{2}{*}{0.000} \\
\hline$\leq 2.05$ & 37 (94.7) & $2(5.3)$ & \\
\hline \multicolumn{4}{|c|}{$\operatorname{MTV} 3\left(\mathrm{~cm}^{3}\right)$} \\
\hline$>1.54$ & $5(41.7)$ & $7(58.3)$ & \multirow[t]{2}{*}{0.000} \\
\hline$\leq 1.54$ & 37 (94.7) & $2(5.3)$ & \\
\hline \multicolumn{4}{|c|}{ MTV3.5 $\left(\mathrm{cm}^{3}\right)$} \\
\hline$>1.22$ & $5(41.7)$ & $7(58.3)$ & \multirow[t]{2}{*}{0.000} \\
\hline$\leq 1.22$ & $37(94.7)$ & $2(5.3)$ & \\
\hline \multicolumn{4}{|c|}{ MTV4 $\left(\mathrm{cm}^{3}\right)$} \\
\hline$>0.61$ & $7(50.0)$ & $7(50.0)$ & 0.001 \\
\hline$\leq 0.61$ & 35 (94.6) & $2(5.4)$ & \\
\hline TLG2.5 & & & \\
\hline$>9.09$ & $6(46.2)$ & $7(53.8)$ & 0.000 \\
\hline$\leq 9.09$ & $36(94.7)$ & $2(5.3)$ & \\
\hline TLG3 & & & \\
\hline$>5.35$ & $7(50.0)$ & $7(50.0)$ & 0.001 \\
\hline$\leq 5.35$ & 35 (94.6) & $2(5.4)$ & \\
\hline TLG3.5 & & & \\
\hline$>4.42$ & $7(50.0)$ & $7(50.0)$ & 0.001 \\
\hline$\leq 4.42$ & 35 (94.6) & $2(5.4)$ & \\
\hline TLG4 & & & \\
\hline$>3.47$ & $7(50.0)$ & $7(50.0)$ & 0.001 \\
\hline$\leq 3.47$ & 35 (94.6) & $2(5.4)$ & \\
\hline
\end{tabular}

Data are expressed as frequency (\%) for categorical variables $C L N$ central lymph node, ETE extrathyroidal extension, $S U V$ standardized uptake value, MTV metabolic tumor volume, $T L G$ total lesion glycolysis 
Table 4 Optimal cutoff values of $\mathrm{SUV}_{\max }$ and volumetric parameters for prediction of the lateral lymph node metastasis

\begin{tabular}{lllllll}
\hline Criteria & Cutoff value & Sensitivity $(95 \%$ CI $)$ & Specificity (95 \% CI) & AUC $(95 \%$ CI $)$ & SE & $P$ value \\
\hline $\begin{array}{lllll}\text { SUV } \\
\text { MTV }\left(\mathrm{cm}^{3}\right)\end{array}$ & 5.91 & $77.9 \%(40.0-97.2 \%)$ & $69.1 \%(52.9-82.4 \%)$ & $0.716(0.572-0.833)$ & 0.109 & 0.047 \\
MTV2.5 & 2.05 & $77.8 \%(40.0-97.2 \%)$ & $88.1 \%(74.4-96.0 \%)$ & $0.839(0.709-0.927)$ & 0.099 & $<0.001$ \\
MTV3 & 1.54 & $77.8 \%(40.0-97.2 \%)$ & $88.1 \%(74.4-96.0 \%)$ & $0.825(0.693-0.917)$ & 0.104 & 0.002 \\
MTV3.5 & 1.22 & $77.8 \%(40.0-97.2 \%)$ & $88.1 \%(74.4-96.0 \%)$ & $0.815(0.681-0.909)$ & 0.101 & 0.002 \\
MTV4 & 0.61 & $77.8 \%(40.0-97.2 \%)$ & $83.3 \%(68.6-93.0 \%)$ & $0.798(0.662-0.897)$ & 0.101 & 0.003 \\
TLG & & $77.8 \%(40.0-97.2 \%)$ & $85.7 \%(71.5-94.6 \%)$ & $0.815(0.681-0.910)$ & 0.099 & 0.002 \\
TLG2.5 & 9.09 & $77.8 \%(40.0-97.2 \%)$ & $83.3 \%(68.6-93.0 \%)$ & $0.802(0.666-0.900)$ & 0.103 & 0.003 \\
TLG3 & 5.35 & $77.8 \%(40.0-97.2 \%)$ & $83.3 \%(68.6-93.0 \%)$ & $0.802(0.666-0.900)$ & 0.101 & 0.003 \\
TLG3.5 & 4.42 & $77.8 \%(40.0-97.2 \%)$ & $83.3 \%(68.6-93.0 \%)$ & $0.790(0.653-0.891)$ & 0.099 & 0.004 \\
TLG4 & 3.47 & & & & & \\
\hline
\end{tabular}

$A U C$ area under curve, $C I$ confidence interval, $M T V$ metabolic tumor volume $\left(\mathrm{cm}^{3}\right), S E$ standard error, $T L G$ total lesion glycolysis

\section{Prediction of lateral lymph node metastasis from semi-quantitative metabolic parameters of primary thyroid carcinoma}

ROC analyses were performed to determine the optimal cutoff values of semi-quantitative indices for prediction of pathologic lateral LN involvement of thyroid carcinoma. Table 4 and Fig. 2 summarize the results of ROC analyses. The AUC for predicting lateral LNM with $\mathrm{SUV}_{\max }$ was $0.716(P=0.047)$. The AUCs for predicting lateral LNM with MTV calculated at 2.5, 3.0, 3.5, and 4.0 of fixed $\mathrm{SUV}_{\max }$ threshold were $0.839 \quad(P<0.001), \quad 0.825$ $(P=0.002), 0.815(P=0.002)$, and $0.798(P=0.003)$, respectively. The AUCs for predicting lateral LNM with TLG calculated at 2.5, 3.0, 3.5, and 4.0 of fixed $\mathrm{SUV}_{\text {mean }}$ threshold were $0.815(P=0.002), 0.802(P=0.003)$, $0.802(P=0.003)$, and $0.790(P=0.004)$, respectively. Therefore, we used the MTV2.5 and TLG2.5 showing the lowest $\mathrm{P}$ value and the highest AUC for prediction of lateral LNM (Fig. 2).

\section{Comparison of ROC curve analysis}

Table 5 shows the results of pairwise comparison of ROC analyses of semi-quantitative parameters of ${ }^{18}$ F-FDG PET/ CT for prediction of pathologic lateral LNM of thyroid carcinoma. Compared with $\mathrm{SUV}_{\max }$, the MTV2.5 and TLG2.5 showed the statistical differences for the prediction of pathologic lateral LNM of thyroid carcinoma (MTV vs. $\mathrm{SUV}_{\max } P<0.0125$, TLG vs. $\mathrm{SUV}_{\max } P=0.0133$ ). However, there was no significant difference between MTV2.5 and TLG2.5 $(P=0.1298)$.

\section{Discussion}

The value of preoperative F18-FDG PET/CT in PTC remains unclear. As one of the candidate predictors for the evaluation of lateral LNM and local invasiveness of DTC, we evaluated whether the volume-based parameters of ${ }^{18} \mathrm{~F}$ FDG PET/CT imaging could predict pathologic lateral LNM and aggressiveness in patients with incidentally detected DTC in this study. The current study demonstrated that the $\mathrm{SUV}_{\max }$, MTV, and TLG measured by ${ }^{18} \mathrm{~F}-\mathrm{FDG}$ PET/CT had significant association with lateral LNM in patients with incidentally detected DTC. The MTV and TLG had significant value for the prediction of pathologic lateral LNM in comparison with $\mathrm{SUV}_{\max }$.

It is well known that PTC generally shows an indolent disease course. However, PTC frequently metastasizes or recurs to regional $\mathrm{LN}$. Metastasis to lateral $\mathrm{LN}$ is associated with a poor prognosis [13-15]. Thus, imaging the neck before surgery for thyroid carcinoma is necessary to determine the appropriate extent of surgical resection. In addition, it is important to identify patients who are at risk of developing distant metastasis or recurrence that necessitate more aggressive surgical treatment or more intensive radioactive iodine (RAI) therapy. In this regard, ${ }^{18} \mathrm{~F}-\mathrm{FDG}$ PET/CT scans provide anatomical as well as prognostic information that can identify the patients at higher risk of recurrence and metastatic disease $[16,17]$. However, ${ }^{18}$ F-FDG PET/CT is not routinely used in daily clinical practice in characterizing thyroid nodules and preoperative investigation of thyroid carcinomas. It is well known that US is useful in the preoperative assessment of cervical LN metastasis in patients with DTC. Very recently, Lee et al. [10] demonstrated that 

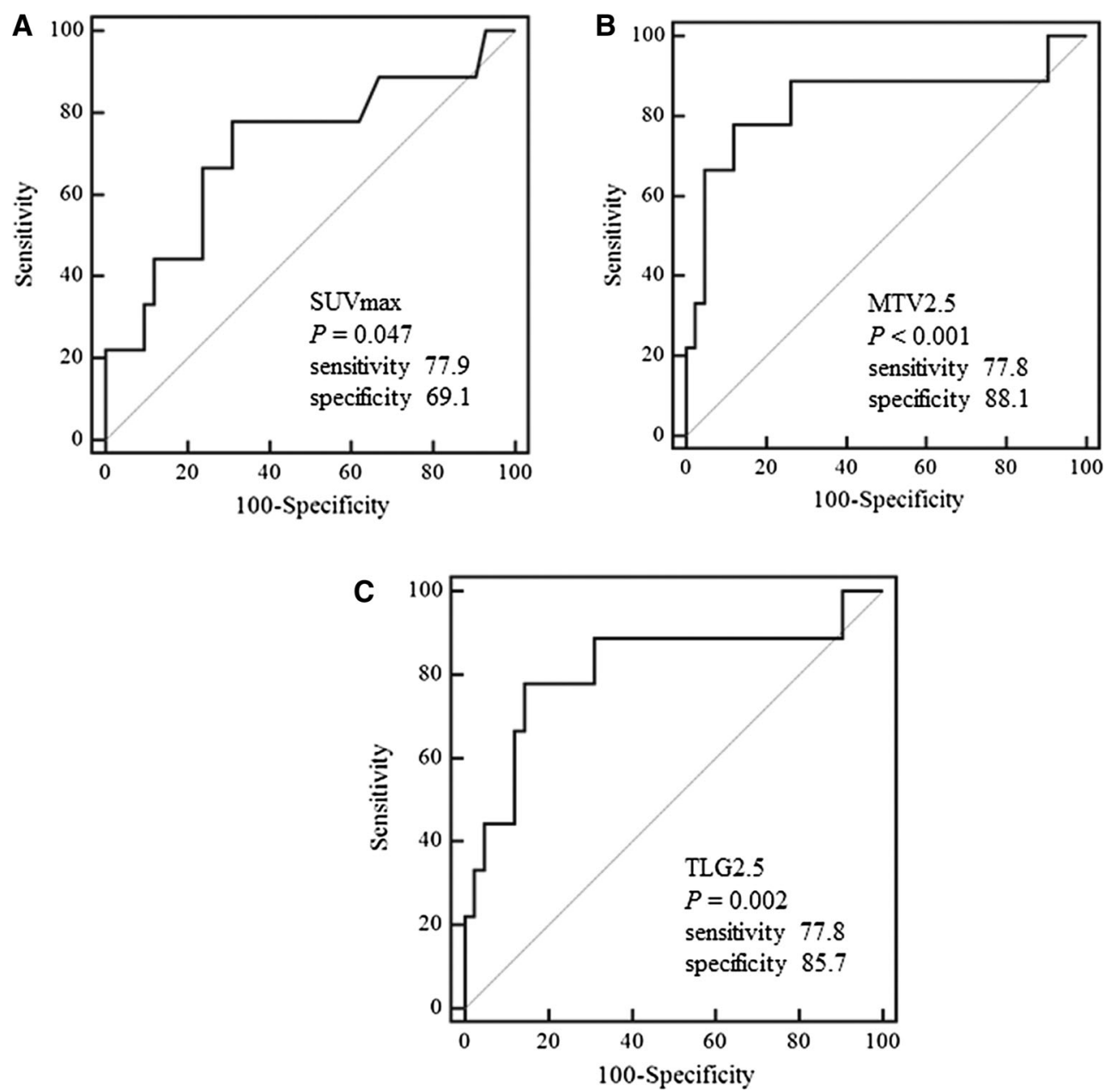

Fig. 2 Receiver operating characteristic curves (ROC) using a $\mathrm{SUV}_{\max }$, b metabolic tumor volume (MTV), and c total lesion glycolysis (TLG) to predict lateral lymph node metastasis

Table 5 Pairwise comparison of ROC curves of semi-quantitative indices of ${ }^{18} \mathrm{~F}$-FDG PET-CT for the lateral lymph node metastasis

\begin{tabular}{lll}
\hline & MTV2.5 & TLG2.5 \\
\hline SUV $_{\max }$ & & \\
DBA & 0.123 & 0.0992 \\
SE & 0.0493 & 0.0401 \\
$95 \%$ CI & $0.0264-0.220$ & $0.0207-0.178$ \\
$P$ & 0.0125 & 0.0133 \\
MTV2.5 & & \\
DBA & & 0.0238 \\
SE & & 0.0157 \\
$95 \%$ CI & & -0.0069 to 0.0546 \\
$P$ & & 0.1298
\end{tabular}

$D B A$ difference between areas, $C I$ confidence interval, $M T V$ metabolic tumor volume, $S E$ standard error, $S U V$ standardized uptake value, $T L G$ total lesion glycolysis preoperative US might be helpful in detecting nodal metastases in PTC patients.

The value of FDG uptake in preoperative risk stratification remains controversial. In this study, $25.5 \%(13 / 51)$ of patients showed FDG uptake in lateral LN. Increased FDG uptake of lateral LN metastasis showed low sensitivity and PPV but high specificity and NPV for pathologically proven lateral LNM. Although three patients who had US-guided FNA-confirmed lateral LN metastasis did not show FDG uptake in lateral LNs on F18-FDG PET/CT, preoperative FDG negativity of lateral LNs suggested low possibility in lateral LNM in patients with DTC. Therefore, both preoperative F18-FDG PET-CT and US may improve sensitivity and specificity of prediction of lateral LNM in incidentally detected thyroid carcinoma.

A previous study showed that the SUV of thyroid carcinoma alone was not predictive of the presence of ETE, 
LNM, or the multiplicity of PTMC [18]. In contrast, Yun et al. [19] reported that visual FDG positivity is a potential new risk factor that may be useful for preoperative risk stratification of PTMC. Gender, age, and tumor size showed a correlation with either ETE or central LNM, while visually discernible FDG uptake was associated with a higher prevalence of ETE and central LNM. However, in the current study, all parameters measured by ${ }^{18} \mathrm{~F}-\mathrm{FDG}$ PET/CT had no value in prediction of central LNM, ETE, and multifocality except lateral LNM. In particular, the MTV and TLG were better predictors of lateral LNM than $\mathrm{SUV}_{\max }$. When all patients were classified into two groups according to the presence of lateral LNM, tumor size greater than $1 \mathrm{~cm}$ and central LNM were significantly associated with lateral LNM. However, other clinicopathologic characteristics including age, gender, ETE, and multifocality showed no significant difference between the two groups.

It is well established that ETE is an important adverse prognostic factor for patients with PTC [20, 21]. However, recent studies reported that microscopic ETE might not be a prognostic factor to predict tumor recurrence in patients with PTC [22, 23]. Thus, it is unclear that microscopic ETE is associated with poor prognostic factor. No significant predictive value of volume-based parameters for ETE observed in the current study is thought to be that most ETE in our patients (22/23) is minimal microscopic extrathyroidal invasion into the strap muscle.

It is still unclear whether or not FDG positivity of DTC in the preoperative ${ }^{18} \mathrm{~F}$-FDG PET-CT indicates tumor aggressiveness. The reason of the discrepancy between previous studies [4-7] and our findings in DTC could be that tumor size of incidentally detected PTC was relatively small compared to other cancers. The partial volume effect (PVE) can have a major effect on the measurement of tumor uptake with PET. The PVE may affect MTV or TLG [24]. The PVE strongly depends on the size of the tumor. The smaller the tumor, the greater the underestimation of the uptake value [25]. In addition, the accuracy of PETbased automatic or semiautomatic delineation methods can be affected by many factors, including image resolution and reconstruction settings [26, 27].

This study has some limitations. First, all patients with focal FDG-avid thyroid incidentaloma did not underwent FNAB and surgery to confirm malignancy due to underlying cancer and general condition. Second, the limited sample size of surgically confirmed DTC and the retrospective design are also limitations of this study. Third, a large proportion of the patients studied in this series have had former cancer treatment which might have influenced measured metabolic parameters. Despite these limitations, we have, for the first time, found the clinical implication of volume-based parameters of ${ }^{18} \mathrm{~F}-\mathrm{FDG}$ PET/CT in preoperative risk stratification of incidentally detected thyroid cancers.

In conclusion, this study has shown that volume-based PET functional parameters such as TLG and MTV can provide metabolic information of incidentally detected PTC. The TLG and MTV had significant value for the prediction of lateral LNM in comparison with $\mathrm{SUV}_{\max }$. These results suggest that higher MTV and TLG can be potential new risk factors for preoperative risk stratification. Therefore, thyroid cancer with high MTV and TLG should be closely examined for LNM by preoperative neck US. Future large prospective studies will provide greater clarity regarding the value of these parameters of preoperative ${ }^{18}$ F-FDG PET-CT in patients with incidentally detected DTC.

\section{Complaince with ethical standards}

Conflict of interest The authors declare that there is no conflict of interest.

\section{References}

1. Larson SM, Erdi Y, Akhurst T, Mazumdar M, Macapinlac HA, Finn RD, et al. Tumor treatment response based on visual and quantitative changes in global tumor glycolysis using PET-FDG imaging. The visual response score and the change in total lesion glycolysis. Clin Positron Imaging. 1999;2(3):159-71.

2. Erdi YE, Macapinlac H, Rosenzweig KE, Humm JL, Larson SM, Erdi AK, et al. Use of PET to monitor the response of lung cancer to radiation treatment. Eur J Nucl Med. 2000;27(7):861-6.

3. Zhu D, Ma T, Niu Z, Zheng J, Han A, Zhao S, et al. Prognostic significance of metabolic parameters measured by ${ }^{18}$ F-fluorodeoxyglucose positron emission tomography/computed tomography in patients with small cell lung cancer. Lung Cancer. 2011;73(3):332-7.

4. Dibble EH, Alvarez AC, Truong MT, Mercier G, Cook EF, Subramaniam RM. 18F-FDG metabolic tumor volume and total glycolytic activity of oral cavity and oropharyngeal squamous cell cancer: adding value to clinical staging. J Nucl Med. 2012;53(5):709-15.

5. Hs I. Kim SJ, Kim IJ, Kim K. Predictive value of metabolic tumor volume measured by 18F-FDG PET for regional lymph node status in patients with esophageal cancer. Clin Nucl Med. 2012;37(5):442-6.

6. Romesser PB, Qureshi MM, Shah BA, Chatburn LT, Jalisi S, Devaiah AK, et al. Superior prognostic utility of gross and metabolic tumor volume compared to standardized uptake value using PET/CT in head and neck squamous cell carcinoma patients treated with intensity-modulated radiotherapy. Ann Nucl Med. 2012;26(7):527-34.

7. Van de Wiele C, Kruse V, Smeets P, Sathekge M, Maes A. Predictive and prognostic value of metabolic tumour volume and total lesion glycolysis in solid tumours. Eur J Nucl Med Mol Imaging. 2013;40(2):290-301.

8. Stack BC Jr, Ferris RL, Goldenberg D, Haymart M, Shaha A, Sheth S, et al. American Thyroid Association consensus review and statement regarding the anatomy, terminology, and rationale for lateral neck dissection in differentiated thyroid cancer. Thyroid. 2012;22(5):501-8. 
9. Lee DW, Ji YB, Sung ES, Park JS, Lee YJ, Park DW, et al. Roles of ultrasonography and computed tomography in the surgical management of cervical lymph node metastases in papillary thyroid carcinoma. Eur J Surg Oncol. 2013;39(2):191-6.

10. Lee YJ, Kim DW, Kyoung Park H, Kim DH, Jung SJ, Oh M, et al. Pre-operative ultrasound diagnosis of nodal metastasis in papillary thyroid carcinoma patients according to nodal compartment. Ultrasound Med Biol. 2015;41(5):1294-300.

11. Yasuda S, Shohtsu A, Ide M, Takagi S, Takahashi W, Suzuki Y, et al. Chronic thyroiditis: diffuse uptake of FDG at PET. Radiology. 1998;207(3):775-8.

12. Kim BH, Kim SJ, Kim H, Jeon YK, Kim SS, Kim IJ, et al. Diagnostic value of metabolic tumor volume assessed by ${ }^{18} \mathrm{~F}$ FDG PET/CT added to $\mathrm{SUV}_{\max }$ for characterization of thyroid 18F-FDG incidentaloma. Nucl Med Commun. 2013;34(9): $868-76$.

13. Mazzaferri EL. Long-term outcome of patients with differentiated thyroid carcinoma: effect of therapy. Endocr Pract. 2000;6(6):469-76.

14. Grebe SK, Hay ID. Thyroid cancer nodal metastases: biologic significance and therapeutic considerations. Surg Oncol Clin N Am. 1996;5(1):3-43.

15. Kim TY, Kim WG, Kim WB, Shong YK. Current status and future perspectives in differentiated thyroid cancer. Endocrinol Metab (Seoul). 2014;29(3):217-25.

16. Shammas A, Degirmenci B, Mountz JM, McCook BM, Branstetter B, Bencherif B, et al. ${ }^{18}$ F-FDG PET/CT in patients with suspected recurrent or metastatic well-differentiated thyroid cancer. J Nucl Med. 2007;48(2):221-6.

17. Wang W, Larson SM, Fazzari M. Prognostic value of $\left[{ }^{18} \mathrm{~F}\right]-$ fluorodeoxy glucose positron emission tomographic scanning in patients with thyroid cancer. J Clin Endocrinol Metab. 2000;85(3):1107-13.

18. Jeong HS, Chung M, Baek CH, Ko YH, Choi JY, Son YI. Can [18F]-fluorodeoxyglucose standardized uptake values of PET imaging predict pathologic extrathyroid invasion of thyroid papillary microcarcinomas? Laryngoscope. 2006;116(12):2133-7.

19. Yun M, Noh TW, Cho A, Choi YJ, Hong SW, Park CS, et al. Visually discernible $\left[{ }^{18} \mathrm{~F}\right]$ fluorodeoxyglucose uptake in papillary thyroid microcarcinoma: a potential new risk factor. J Clin Endocrinol Metab. 2010;95(7):3182-8.

20. Ortiz S, Rodríguez JM, Soria T, Pérez-Flores D, Piñero A, Moreno J, et al. Extrathyroid spread in papillary carcinoma of the thyroid: clinicopathological and prognostic study. Otolaryngol Head Neck Surg. 2001;124(3):261-5.

21. Radowsky JS, Howard RS, Burch HB, Stojadinovic A. Impact of degree of extra-thyroidal extension of disease on papillary thyroid cancer outcome. Thyroid. 2014;24(2):241-4.

22. Arora N, Turbendian HK, Scognamiglio T, Wagner PL, Goldsmith SJ, Zarnegar R, et al. Extrathyroidal extension is not all equal: implications of macroscopic versus microscopic extent in papillary thyroid carcinoma. Surgery. 2008;144(6):942-7.

23. Kim JM, Lee YY, Choi CW, Lim SM, Lee S, Cho SY, et al. The clinical importance of minimal extrathyroidal extension on tumor recurrence in patients with papillary thyroid carcinoma. Endocrinol Metab. 2010;25(4):340-6.

24. Hatt M, Le Pogam A, Visvikis D, Pradier O. Cheze Le Rest C. Impact of partial-volume effect correction on the predictive and prognostic value of baseline ${ }^{18} \mathrm{~F}-\mathrm{FDG}$ PET images in esophageal cancer. J Nucl Med. 2012;53(1):12-20.

25. Soret M, Bacharach SL, Buvat I. Partial-volume effect in PET tumor imaging. J Nucl Med. 2007;48(6):932-45.

26. Geets X, Lee JA, Bol A, Lonneux M, Grégoire V. A gradient-based method for segmenting FDG-PET images: methodology and validation. Eur J Nucl Med Mol Imaging. 2007;34(9):1427-38.

27. Schaefer A, Kremp S, Hellwig D, Rübe C, Kirsch CM, Nestle U. A contrast-oriented algorithm for FDG-PET-based delineation of tumour volumes for the radiotherapy of lung cancer: derivation from phantom measurements and validation in patient data. Eur $\mathbf{J}$ Nucl Med Mol Imaging. 2008;35(11):1989-99. 